\title{
Fiber-based ultraviolet frequency sweep laser
}

\author{
Xiong Wan ${ }^{1, a^{*}}$ and Pengxi Liü, b \\ ${ }^{1}$ Key Laboratory of Space Active Opto-Electronics Technology, Shanghai Institute of Technical \\ Physics of the Chinese Academy of Sciences, Shanghai 200083, China \\ ${ }^{2}$ Key Laboratory of Space Active Opto-Electronics Technology, Shanghai Institute of Technical \\ Physics of the Chinese Academy of Sciences, Shanghai 200083, China \\ awanxiong1@126.com, blpx.ustc@gmail.com
}

Keywords: Resonance Raman; Ultraviolet Raman; frequency sweep; laser

Abstract. A resonance Raman detection system (RRDS) is proposed in this paper. The system is composed of a miniature ultraviolet frequency sweep laser (UFSL) and a controller and data analyzer (CDA). The UFSL consists of a pulse semiconductor laser (PSL) module, a fiber laser module (FLM), and an ultraviolet frequency sweep module (UFSM). Among the molecules of the tested object, those molecules, whose resonance Raman excitation wavelength equals the ultraviolet sweep wavelength and Stoke Raman spectral intensity will then increase to $10^{4}$ to $10^{6}$ times due to the resonance Raman effect, can be detected in the case of extremely tiny content. The advantages of the RRDS include integral structure of electronics and optical fibers, miniature of UFSL, fast regulation of the ultraviolet laser wavelengths, elimination of fluorescence interference.

\section{Introduction}

When monochromatic light illuminates objects, most of the light will be reflected in the original direction, meanwhile small part of the light being scattered from different angles. The predominant scattering is Rayleigh scattering[1], which has the same frequency as the incident light. In addition, some scattered light belongs to Raman scattering[2], which has a negative or positive frequency shift relative to the incident light. Intensities and frequency shifts of Raman scattering are related to vibration or rotation energy levels of molecules of detected objects[3]. Hence, Raman spectroscopy can be used to obtain the information on molecular vibration or rotation and then study the structure of molecules.

There are two drawbacks, namely weakness of signals and being susceptible to fluorescence interference, in conventional detection techniques with laser Raman[4]. Ultraviolet Raman spectroscopic (URS) techniques[5-7] are adopted to eliminate the fluorescence interference. In URS techniques, targets are excited with lasers of ultraviolet wavelengths, and the excited Stokes Raman scatterings are also located in ultraviolet band that is separated from the visible band of fluorescence.

Resonance Raman spectroscopy (RRS)[8-10] can enhance Raman scattering signals dramatically when the excitation laser frequency is close or equivalent to the electronic transition frequency of probed molecules, causing strong absorption or resonance and leading to millions of times signal enhancement for some Raman band intensities of the molecules. Therefore, RRS is beneficial for Raman analyses. Ultraviolet resonance Raman spectroscopy (URRS), combining URS with RRS, can detect the distribution of a certain molecule with a high sensitivity. However, URRS can not realize high sensitive detection for multiple molecules simultaneously.

If excitation lasers with a frequency sweep technique[11] are applied in the ultraviolet band, multiple molecules will be excited resonantly, and then high sensitive detection for multiple molecules can be realized. 


\section{Resonance Raman detection system}

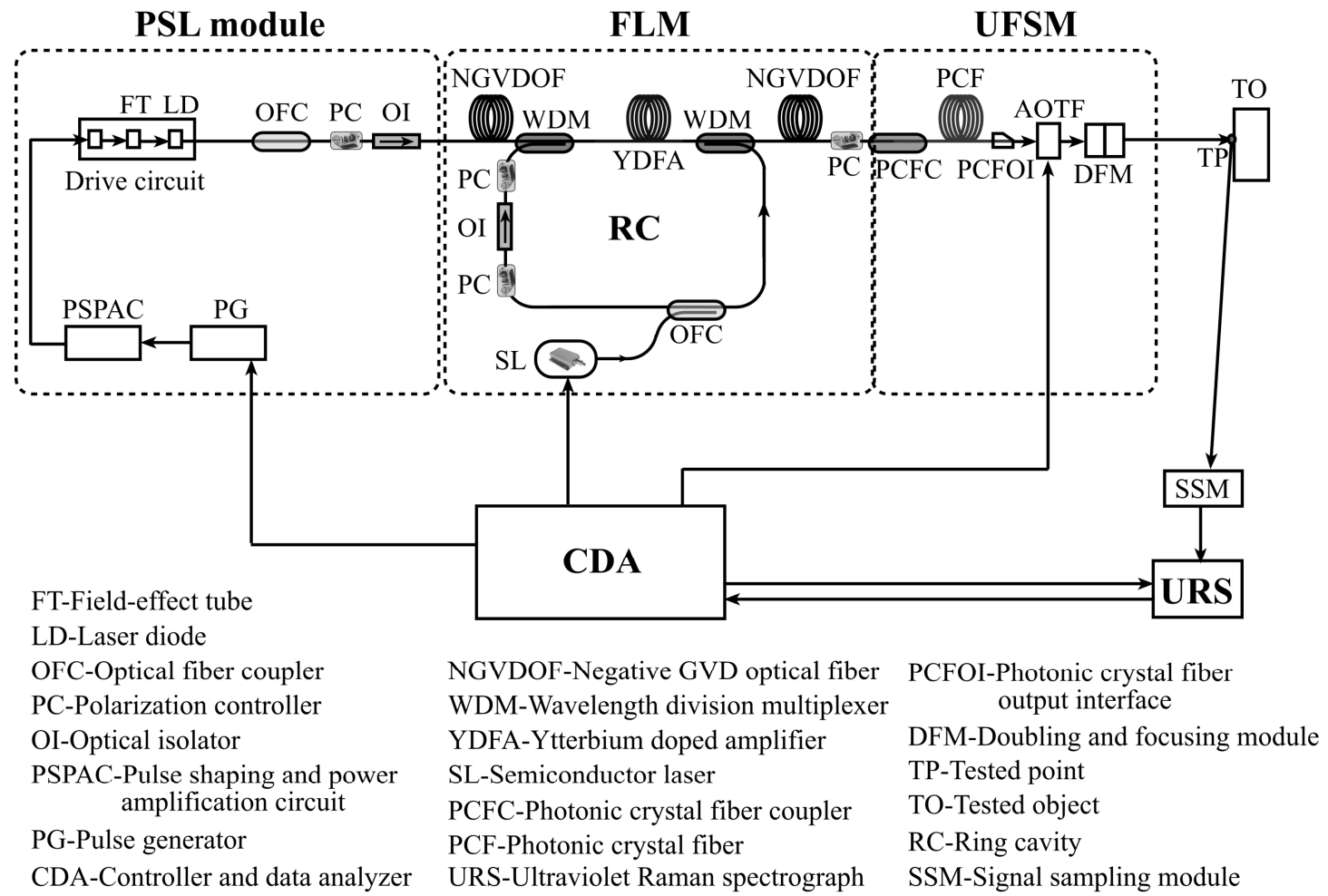

Fig. 1. Layout of the resonance Raman detection system (RRDS)

A resonance Raman detection system (RRDS), shown in Fig. 1, is proposed in this paper. RRDS is based on a miniature ultraviolet frequency sweep laser (UFSL), and consists of the UFSL and its corresponding controller and data analyzer (CDA). The UFSL comprises a pulse semiconductor laser (PSL) module, a fiber laser module (FLM), and an ultraviolet frequency sweep module (UFSM). The PSL module consists of a FPGA pulse generator, a pulse shaping and power amplification circuit (PSPAC), a PSL and its drive circuit, an optical fiber coupler, a polarization controller, and an optical isolator. The fiber laser is pumped by a pulse seed light generated with the PSL. The FLM mainly comprises a semiconductor laser, a fiber ring cavity, and two optical fibers of negative group velocity delay (GVD). The function of FLM is to amplify and lock mode of the pulse seed light transmitted from PSL. The UFSM is mainly composed of a photonic crystal fiber (PCF), an acoustic optical tunable filter (AOTF), a frequency doubling module, and a focusing module. A super-continuum pulse laser beam is generated through UFSM, and then the frequency of the laser beam is doubled to ultra violet band. Finally the ultra violet laser beam will be swept and focused to output.

The CDA, consisting of a control circuit and an analysis software, is used to control the AOTF, the PSL, the FPGA pulse generator, and a ultraviolet Raman spectrograph (URS). Then the spectroscopic data outputted from the URS will be received and analyzed with the CDA.

\section{Detection of frequency sweep resonance Raman spectroscopy}

The detection of frequency sweep resonance Raman spectroscopy includes the following steps:

a. The CDA initiates AOTF and selects the initial wavelength $\lambda_{1}$ of frequency sweep, and initiates FPGA pulse generator and sets its frequency to $80 \mathrm{MHz}$.

b. The CDA initiates PSL. The electric pulse generated by FPGA is shaped and amplified via the PSPAC, and drive the PSL to emit a pulse seed laser beam with frequency of $80 \mathrm{MHz}$ and pulse width of several nano-seconds. This seed laser beam enters the optical fiber via the fiber coupler, and transmits into the FLM via the polarization controller and the optical isolator. The function of the optical isolator is to eliminate the echo interference from the FLM. 
c. The continuous laser beam emitted with the fiber laser in FLM separated in two directions and enters the fiber ring cavity. Then the two beams pump an ytterbium doped fiber amplifier (YDFA) via two wavelength division multiplexers from two directions. The seed laser beam outputted from the PSL is amplified by the YDFA via the fiber of negative GVD. The pulse of seed laser beam is compressed, after passing the fiber of negative GVD, and then is stretched after passes the YDFA that has a positive GVD. Afterward, the pulse is compressed again after passing the second fiber of negative GVD, generating a chirp compensation in the ring cavity. The pulse becomes a femto-second pulse due to the self mode locking mechanism in these nonlinear birefraction fibers, propagates in the ring cavity along one direction, and then outputs into the UFSM via a polarization controller.

d. The femto-second laser pulse outputted from FLM enters into the PCF via a PCF coupler, and turns into a super-continuum laser pulse.

e. The super-continuum laser pulse passes through the AOTF, and turns into a femto-second laser pulse of wavelength $\lambda_{1}$. The frequency doubling module comprises two frequency doubling crystals that can realize fourth harmonic conversion. The focusing module can achieve the focus of ultraviolet laser. After the laser pulse outputted from AOTF enters the frequency doubling and focusing module, it will turn into a femto-second laser pulse of wavelength $\lambda_{1} / 4$ and frequency $80 \mathrm{MHz}$, and then be focused on the tested object.

f. The CDA initiates the URS, which then analyzes the ultraviolet Raman signals emitted from the focusing point of the tested object. Among the molecules of the tested object, those molecules, whose resonance Raman excitation wavelength equals $\lambda_{1} / 4$ and Stoke Raman spectral intensity will then increase to $10^{4}$ to $10^{6}$ times due to the resonance Raman effect, can be detected in the case of extremely tiny content.

g. The CDA chooses the wavelength of AOTF as $\lambda_{2}=\lambda_{1}+\Delta \lambda_{1}$, here being the step of frequency sweep. The super-continuum laser pulse, outputted from PCF, passes through the AOTF, and turns into a femto-second laser pulse of wavelength $\lambda_{2}$. After the laser pulse outputted from AOTF enters the frequency doubling and focusing module, it will turn into a femto-second laser pulse of wavelength $\lambda_{2} / 4$ and frequency $80 \mathrm{MHz}$, and then be focused on the tested object. those molecules, whose resonance Raman excitation wavelength equals $\lambda_{2} / 4$ and Stoke Raman spectral intensity will then increase to $10^{4}$ to $10^{6}$ times due to the resonance Raman effect, can be detected in the case of extremely tiny content.

h. Repeat the step g, till all the molecules have been detected.

\section{Discussion}

We propose a resonance Raman detection system (RRDS) here and introduce its structure in detail. Then, the procedure of RRDS is listed. The RRDS has an integral structure of electronics and optical fibers and a miniature of UFSL. We can easily regulate the ultraviolet laser wavelengths and eliminate the fluorescence interference with RRDS. Hence, RRDS has an application prospective in optical field.

\section{Acknowledgements}

This work was supported by National High-tech Development Plan 863 under Grant 2015AA021103, Chinese Natural Science Fund under Grant 81260225, Fund of CAS key Laboratory under Grant CXJJ-14-S134, and Project of "Hundred Talents Plan" of CAS.

\section{References}

[1] Y. Harada, T. Asakura, Radiation forces on a dielectric sphere in the Rayleigh scattering regime, Opt. Commun. 124.5-6(1996)529-541.

[2] E.J. Heller, R. Sundberg, D. Tannor, Simple aspects of Raman scattering, J. Phys. Chem. 86.10(1982)1822-1833. 
[3] W. Zinth, W. Kaiser, Frequency shifts in stimulated Raman scattering, Opt. Commun. 32.3(1980)507-511.

[4] M.R. Kagan, R.L. Mccreery, A. Chem, Reduction of Fluorescence Interference in Raman Spectroscopy via Analyte Adsorption on Graphitic Carbon, Anal. Chem. 66.23(1994)4159-4165.

[5] S.P.A. Fodor, R.A. Copeland, C.A.Grygon, et al., Deep-ultraviolet Raman excitation profiles and vibronic scattering mechanisms of phenylalanine, tyrosine, and tryptophan, J. Am. Chem. Soc. 111.15(1989)5509-5518.

[6] R.A. Copeland, T.G. Spiro, Secondary structure determination in proteins from deep (192-223-nm) ultraviolet Raman spectroscopy, Biochemistry. 26.8(1987)2134-2139.

[7] C. Li, P.C. Stair, Ultraviolet Raman spectroscopy characterization of coke formation in zeolites, Catal. Today. 33(1997)353-360.

[8] R. Saito, A. Gruneis, G.G. Samsonidze, et al., Double resonance Raman spectroscopy of single-wall carbon nanotubes, New J. Phys. 5.23(2003)2032-2035.

[9] R.S. Czernuszewicz, Y.L. Xiao, T.G. Spiro, Nickel octaethylporphyrin ruffling dynamics from resonance Raman spectroscopy, J. Am. Chem. Soc. 111.18(1989)7024-7031.

[10] A.R. Oseroff, R.H. Callender, Resonance Raman spectroscopy of rhodopsin in retinal disk membranes, Biochemistry. 13.20(1974)4243-4248.

[11] T.J. Ahn, D.Y. Kim, Analysis of nonlinear frequency sweep in high-speed tunable laser sources using a self-homodyne measurement and Hilbert transformation, Appl. Opt. 46.13(2007)2394-2400. 